Review began 08/19/2021 Review ended 09/11/2021 Published 09/22/2021

๑) Copyright 2021

Booher et al. This is an open access article distributed under the terms of the Creative Commons Attribution License CC-BY 4.0. which permits unrestricted use, distribution, and reproduction in any medium, provided the original author and source are credited.

\section{Comparison of Three Groups of Patients Having Low Dose Rate Prostate Brachytherapy: Prostate- Specific Antigen Failure and Overall Survival}

Jacquelyn Booher ${ }^{1}$, Peter Domenig ${ }^{1}$, Benjamin Goldman ${ }^{1}$, Todd Campbell ${ }^{2}$, Kyle Verdecchia ${ }^{3}$, Judith A. Boura $^{4}$, Paul J. Chuba ${ }^{5}$

1. Urology, Ascension Macomb-Oakland Hospital, Warren, USA 2. Urology, Ascension Macomb Oakland Hospital, Warren, USA 3. Radiation Oncology, Henry Ford Hospital Detroit, Detroit, USA 4. Research, Ascension MacombOakland Hospital, Warren, USA 5. Radiation Oncology, Ascension Macomb-Oakland Hospital, Warren, USA

Corresponding author: Paul J. Chuba, paul.chuba@ascension.org

\section{Abstract}

Purpose

To examine dosimetric and clinical outcomes for Cs-131 radioactive seed implant compared to Pd-103 and I125 .

\section{Background/Significance}

Cs-131 is a novel isotope with relatively short half-life (9.7 days) that may have clinical advantages in seed implant treatments of prostate cancers. There may be a shorter duration of symptoms and increased PSA control rates.

\section{Methods}

We performed a retrospective study in which clinical and dosimetric outcomes were compared for 186 prostate implants performed over a ten-year time period at three different Ascension hospitals. Isotopes that were used included Cs-131 ( $\mathrm{n}=66$; half-life 9.7 days), I-125 ( $\mathrm{n}=60$; half-life 60 days), and Pd-103 ( $\mathrm{n}=60$; half-life 17 days)

\section{Results}

The implants used standard radiation dosages. These were 145 Gy for I-125 alone or 109 Gy when combined with external beam radiation. In the case of Cs-131 used alone, the dose was $115 \mathrm{~Gy}$ or $85 \mathrm{~Gy}$ when combined with an external beam. For Pd-103, 125 Gy was used for monotherapy and 90 Gy when combined with an external beam. The Cs-131 dosimetry was found to be similar to I-125 and Pd-103 on a quantitative basis. However, there was better homogeneity, and the delivered activity per seed and the number of seeds employed were greater compared to other isotopes. We compared the corrected total source strengths (i.e. normalized to sample mean values) and were able to demonstrate similar distributions for the three isotopes. Dosimetric analysis also suggested there was superior homogeneity with Cs-131. The median PSA value at 60 months was $0.11 \mathrm{ng} / \mathrm{ml}$. There were only a few PSA failures in the three groups of cases, nonetheless, the Cs-131 had the fewest.

\section{Conclusions}

One attractive option for men with early-stage prostate cancer is interstitial brachytherapy. The use of the shorter-acting Cs-131 isotope may be expected to have dose-related side effects that resolve more rapidly. This series suggests a trend for improved PSA control outcomes for Cs-131 patients compared with I-125 and Pd-103.

Categories: Medical Physics, Radiation Oncology, Urology

Keywords: low dose rate prostate brachytherapy, prostate cancer, radiation therapy, radiation physics, seed implant

\section{Introduction}

Prostate cancer is the second most common cause of cancer in men and can be associated with significant morbidity and mortality [1]. One treatment pathway for localized prostate cancer has been permanent prostate seed implant or low dose rate (LDR) brachytherapy [2,3]. This minimally invasive approach has been associated with long-term disease control outcomes that appear to be equivalent to prostatectomy and external beam radiation therapy (EBRT) for low-risk and select intermediate-risk patients [3]. In higher-risk disease, the combination of hormone therapy, external beam radiation, and LDR brachytherapy is associated with superior failure-free and overall survival compared with the combination of hormone therapy and external beam radiation alone $[4,5]$. 
Historically, the two most commonly used isotopes for LDR brachytherapy have been Iodine-125 (I-125) and Palladium-103 (Pd-103) which have a half-life of 61 and 17 days, and energy of $28 \mathrm{KeV}$ and $21 \mathrm{KeV}$ respectively [5]. Cesium-131 is a novel radioisotope with a shorter half-life of 9.7 days and higher energy ( $30.4 \mathrm{KeV})$ which has been suggested to confer possible clinical advantages of increased disease control and shorter duration of symptoms [6-10]. In this report, we examine and compare the dosimetry, PSA failure rates, and overall survival for patients treated with Cs-131, in comparison with I-125, and Pd-103 brachytherapy in a community hospital system.

\section{Materials And Methods}

The data collection interval was from 2007 to 2017 yielding 66 patients with localized prostate cancer who received LDR prostate implant using Cs-131 at Ascension Providence Rochester Hospital, Ascension Macomb-Oakland Hospital, and Ascension St. John Hospital. A comparison cohort consisting of a group of patients treated using I-125 ( $\mathrm{n}=59)$, and Pd-103 ( $\mathrm{n}=61)$ LDR implants having a minimum PSA follow-up of 24 months were selected for evaluation of dosimetry and clinical outcomes. Data was collected as part of an IRB-approved retrospective study.

The radiation doses used for implant were as follows: for I-125 used alone (monotherapy), the dose was 145 $\mathrm{Gy}$, and when combined with external radiation the dose was $109 \mathrm{~Gy}$. The dose for Cs-131 was $115 \mathrm{~Gy}$ when used alone and $85 \mathrm{~Gy}$ when combined with external radiation therapy (1.8 to $2.0 \mathrm{U}$ activity). In the case of Pd-103, the dose when used alone was $125 \mathrm{~Gy}$, or $90 \mathrm{~Gy}$ when combined with external radiation. Postimplant dosimetric information was also collected with parameters reviewed including prostate volume, number of seeds, delivered activity, and total activity. The percent dose received by 90 percent of the prostate (D90), and the percent volume of the prostate receiving 100, 150, and 200 percent of the prescribed dose (V100, V150, and V200) was recorded. The addition of external beam radiation included 45 Gy given in 1.8 Gy daily fractions using 3-dimensional conformal or intensity-modulated radiation therapy methods. For combination therapy, the prostate, seminal vesicles, and (in some patients) the pelvic lymph nodes were treated.

PSA failure and overall survival were calculated for each isotope treatment group. The patients were riskstratified according to the National Comprehensive Cancer Network (NCCN) definitions. Overall survival was based in part on death certificate data obtained from the State of Michigan through the Michigan Cancer Foundation and the Karmanos Cancer Institute. Kaplan-Meier estimates of PSA failure were generated using the 'Phoenix' definition of nadir PSA plus 2 for biochemical failure [11]. Case characteristics were collected in order to determine the D'Amico risk category for each patient. All analyses were completed using SAS for Windows ${ }^{\circledR}$ 9.4, Cary, NC. Kaplan-Meier plots were also completed for each of the 3 different isotope groups for overall survival. The categorical variables were examined with Chi-square tests where appropriate (expected frequency $>5$ in $80 \%$ of cells); otherwise, Fisher's Exact tests were used. Age was compared using the Student's t-test. The remaining continuous variables were analyzed with Wilcoxon rank sum tests since none appeared to be normally distributed for the groups.

\section{Results}

The study included 186 patients who underwent prostate brachytherapy. There were 66 patients with Cs-131 implantation, 59 with I-125, and 61 with Pd-103. The mean pretreatment PSA values for the three groups were Cs-131 $5.73 \mathrm{ng} / \mathrm{ml}, \mathrm{I}-1256.62 \mathrm{ng} / \mathrm{ml}$, and for Pd-130 $8.87 \mathrm{ng} / \mathrm{ml}$. The three implant groups were selected to have adequate follow-up for analysis (median follow-up was 37 months for PSA failure-free survival and 40 months for overall survival). but were relatively well-matched (but not matched in advance) with respect to age, grade, and risk category (Table 1). 


\begin{tabular}{|c|c|c|c|c|c|}
\hline & & Cs-131 (n=66) & $\mathrm{I}-125(n=59)$ & $P d(n=61)$ & $p$-value \\
\hline \multirow{3}{*}{ Age } & Mean (SD) & $68(8.0)$ & $69(7.8)$ & $70(6.0)$ & 0.35 \\
\hline & Median age & 69 & 71 & 70 & \\
\hline & Minimum to Maximum & 48 to 79 & 48 to 81 & 59 to 82 & \\
\hline \multirow[t]{3}{*}{ Race n (\%) } & White & $52(80.0)$ & 49 (83.1) & $42(84)$ & 0.29 \\
\hline & Black & $13(20.0)$ & $9(15.3)$ & $6(12)$ & \\
\hline & Other & $0(0.0)$ & $1(1.7)$ & $2(4)$ & \\
\hline \multirow[t]{4}{*}{ Max Gleason Sum n (\%) } & 6 & $31(47.7)$ & 37 (62.7) & $17(27.9)$ & $0.0007^{*}$ \\
\hline & 7 & $33(50.8)$ & $19(32.2)$ & 39 (63.9) & \\
\hline & 8 & $1(1.2)$ & $2(3.4)$ & $5(8.2)$ & \\
\hline & 9 & $0(0.0)$ & $1(1.7)$ & $0(0.0)$ & \\
\hline \multirow{5}{*}{ Stage } & T1c & $50(78.1)$ & $48(81.4)$ & $17(27.9)$ & $<0.0001^{*}$ \\
\hline & T2a & $11(17.2)$ & $9(15.3)$ & 21 (34.4) & \\
\hline & $\mathrm{T} 2 \mathrm{~b}$ & $0.0(0.0$ & $1(1.7)$ & $13(21.3)$ & \\
\hline & $\mathrm{T} 2 \mathrm{c}$ & $3(4.7)$ & $1(1.7)$ & $8(13.1)$ & \\
\hline & T3 & $0(0.0)$ & $0(0.0)$ & $2(3.3)$ & \\
\hline \multirow[t]{3}{*}{ Pretreatment PSA } & $0-10$ & $51(98.1)$ & $52(88.1)$ & $48(80)$ & $0.03^{*}$ \\
\hline & $10-20$ & $1(1.9)$ & $6(10.2)$ & $9(15)$ & \\
\hline & $>20$ & 0 & 0 & $3(5)$ & \\
\hline \multirow[t]{3}{*}{ D’Amico Risk Category } & Low & 27 & 33 & 14 & $0.04^{*}$ \\
\hline & Intermediate & 26 & 18 & 25 & \\
\hline & High & 1 & 4 & 4 & \\
\hline Combination EBRT & & 25 & 12 & 17 & $0.03^{*}$ \\
\hline Adjuvant Hormone Therapy & & 3 & 11 & 20 & $<0.0001^{*}$ \\
\hline \multirow[t]{2}{*}{ Number of Seeds } & Median & 64 & 74 & 66 & $0.008^{*}$ \\
\hline & Minimum to Maximum & 38 to 98 & 41 to 109 & 32 to 129 & \\
\hline \multirow{2}{*}{ Prostate Volume (cc) } & Median & 33 & 29 & 29 & 0.059 \\
\hline & Minimum to Maximum & 15 to 61 & 15 to 58 & 14 to 61 & \\
\hline
\end{tabular}

\section{TABLE 1: Patient and Tumor Characteristics}

${ }^{*}$ chi-squared

Summary of the pretreatment characteristics for the three groups are shown in Table 1 . The median age at diagnosis for the Cs-131 group was 69 years, similar to the I-125 (71 years) and Pd-103 (70 years). There were significant differences among the three groups with respect to Gleason grade $(\mathrm{p}=0.007)$, clinical stage $(\mathrm{p}<0.0001)$, and pretreatment PSA $(\mathrm{p}=0.03)$ with the highest risk group being Pd-103. Very many patients treated with I-125 and Cs-131 had low risk by the D'Amico category. For patients who underwent Cs-131 prostate brachytherapy, 31 had Gleason Grade 6 (47.7\%), 33 had Gleason Grade 7 (50.8\%), and one patient had Gleason Grade 8 (1.2\%) cancer. Of these patients, 61 had stage T1c or T2a (95.3\%) and 3 had T2c (4.7\%) stage cancer.

For the I-125 patient group, there were 37 patients with Gleason Grade 6 (62.7\%), 19 with Gleason Grade 7 (32.2\%), and 3 patients with Gleason Grade 8 or 9 (5.1\%) cancer. Fifty-seven cases (96.7\%) had stage T1c or T2a, and 2 cases (3.4\%) has stage T2c cancer. For the Pd-103 group, there were 17 patients with Gleason 


\section{Cureus}

Grade 6 (27.9\%), 39 with Gleason Grade 7 (63.9\%), and 5 with Gleason Grade 8 or $9(8.2 \%)$ cancer. There were 38 (62.3\%) stage T1c/T2a, 21 (34.4\%) T2b/T2c, and 2 cases (3.3\%) stage T3 cancer. Combination therapy was utilized in 25 out of the 66 Cs-131 patients with 4500 cGy EBRT. There were 12 of the Pd-103 patients and 17 of the I-125 patients who had combination of 4500 cGy EBRT with brachytherapy.

The median follow-up was 37 months for PSA failure-free survival and 40 months for overall survival. PSA failure rates were compared between the three isotope groups. There were very few PSA failures overall, and zero were noted in the Cs-131 group compared to 11 (9.2\%) PSA failures combined between the I-125 and the Pd-103 groups. The median PSA value at 60 months was $0.11 \mathrm{ng} / \mathrm{ml}$ for all groups. PSA failures were greater with increasing Gleason grade (data not shown). In Figure 1, the PSA failure free survival curves for each of the three groups are shown. The Cs-131 group had improved PSA failure free survival which did appear to reach statistical significance in comparison to each of the other isotopes $(p=0.04)$ but this could not be adjusted by risk category (data not shown). The freedom from PSA failure rate at 36 months was $100 \%$, $95.6 \%$, and $95.2 \%$ for the Cs-131, I-125, and Pd-103 groups respectively.

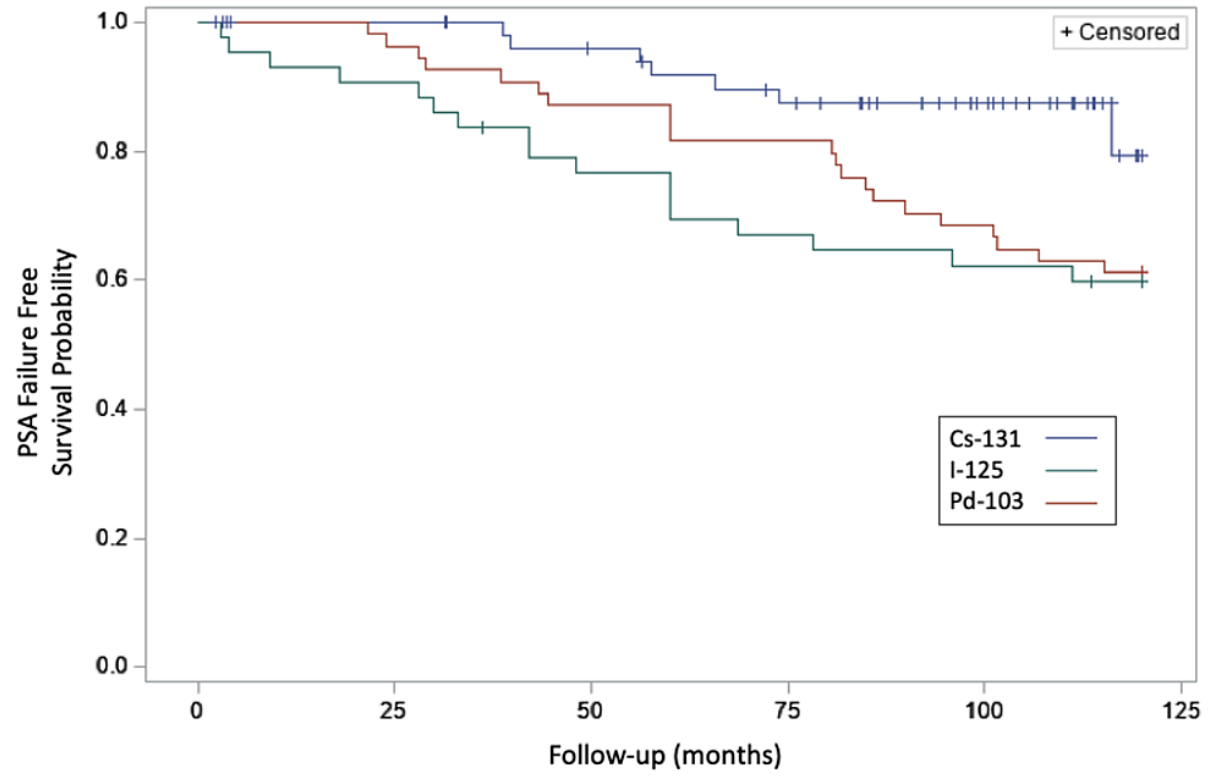

\section{FIGURE 1: PSA Failure Free Survival for Three Groups of Implant}

\section{Patients}

Kaplan Meier curves of PSA failure free survival are shown for three groups of prostate seed implant patients. The upper curve (blue) shows the result for Cs-131 cases, the middle curve (red) shows the result for Pd-103 cases, and the lower curve (green) shows the result for $\mathrm{I}-125$ cases.

The Kaplan Meier curves of overall survival for the three groups are shown in Figure 2. At the conclusion of the data collection interval, a total of 65 patients were deceased, and 87 patients were living. For the patients who received Cs-131, 8/66 (12\%) were deceased. Considering the I-125 and Pd-103 groups, there were 57/120 (48\%) deceased. The 5-year overall survival was $91.7 \%, 88.9 \%, 89.2 \%$ for the Cs-131, I-125, and Pd-103 groups respectively. Although the overall survival of the Cs-131 group was higher compared to the other two groups, statistical significance was not reached $(\mathrm{p}=0.25)$. 


\section{Cureus}

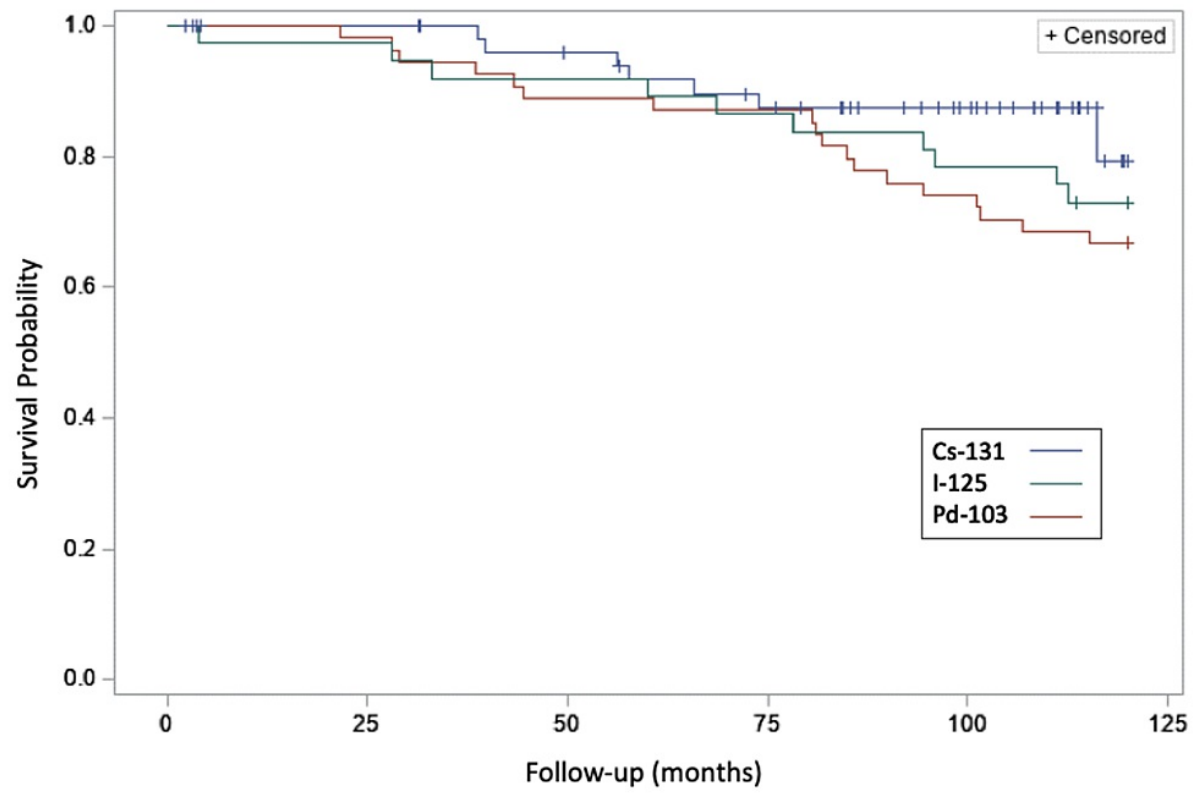

\section{FIGURE 2: Overall Survival for Three Groups of Implant Patients}

Kaplan Meier curves illustrating the overall survival for three groups of patients having seed implants are shown. The upper curve (blue) shows the result for Cs-131 cases, the middle curve (green) shows the result for I-125 cases, and the lower curve (red) shows the result for Pd-103 cases.

With respect to the dosimetric analysis, Cs-131 dosimetry showed better homogeneity (Figure 3). There was greater activity delivered per seed and a greater number of seeds used compared to other isotopes. As shown here, the total source strength (which was normalized to sample mean values) showed a similar distribution for the three isotopes (Figure 3). A comparison of the V150 (mean percent) showed 36\% for I-125 and 27\% for Cs-131 ( $\mathrm{p}<0.0001)$ which suggests that the homogeneity for Cs-131 is better. The percent V200 was $13 \%$ vs $12 \%(\mathrm{p}=0.012$ ) for $\mathrm{I}-125$ versus Cs- 131 . The D90 for Cs- 131 versus I- 125 was $98 \%$ versus $92 \%$, respectively $(\mathrm{p}<0.001)$. As expected, the total source strength was related to prostate volume for all the isotopes showing similar treatment planning (data not shown). 


\section{Cureus}

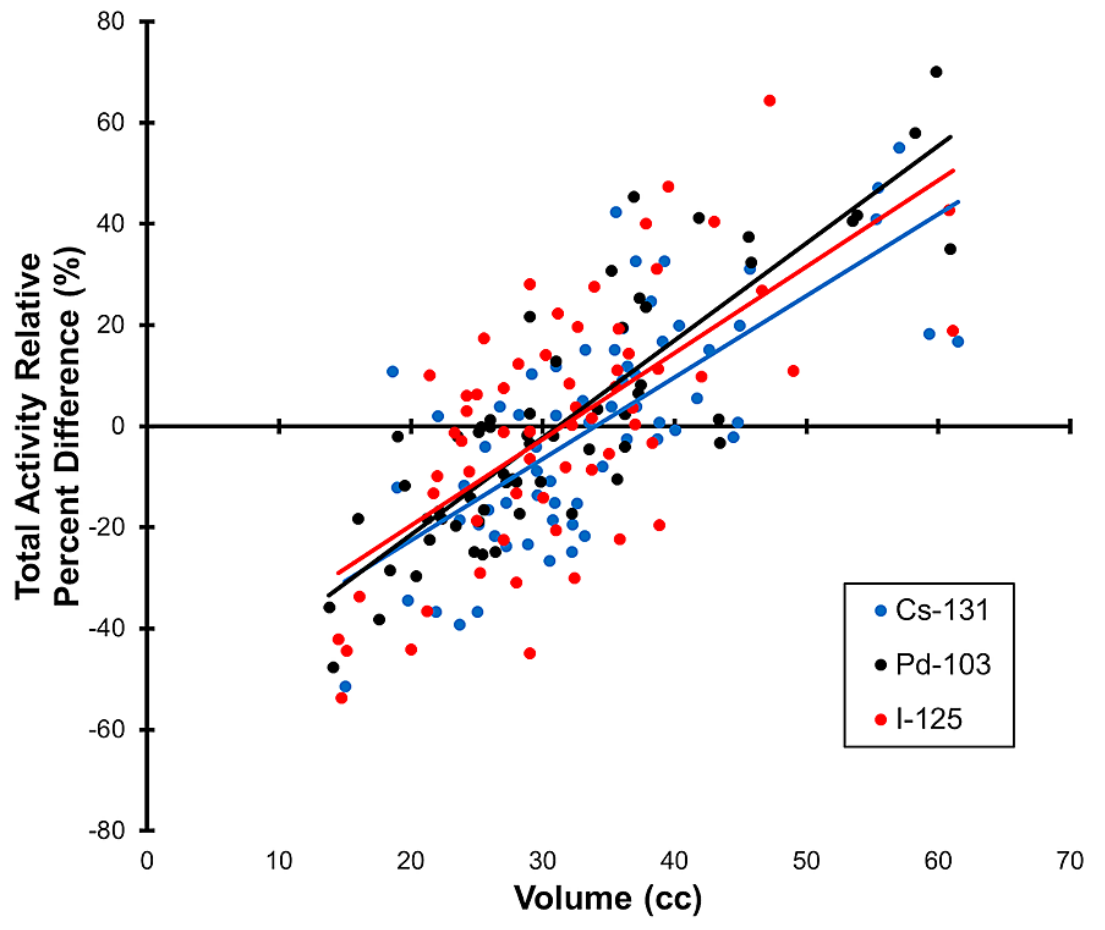

\section{FIGURE 3: Dosimetric Analysis of Prostate Seed Implants with Three}

Different Isotopes

Dosimetry for individual seed implants is illustrated by plotting total activity versus implant volume. The three groups compared are Cs-131 (blue), Pd-103 (black) and l-125 (red).

\section{Discussion}

The Cs-131 implant for prostate cancer was FDA approved in 2003 and to date, there are just a few reports describing long-term outcomes. As the utilization of this treatment option increases, it is important that safety and efficacy, as well as dosimetric equivalence, is studied in comparison to standard therapy. This will facilitate Cs-131 brachytherapy as an option available to a broader patient population outside of large and academic institutions.

The proposed clinical advantages for the use of Cs-131 are related to its energy and half-life [8-10]. In particular, the shorter half-life might be expected to limit the duration of acute urinary morbidity and may be more effective at targeting mitotically active cancer cells. This is because an isotope with shorter half-life may theoretically be more effective in killing higher grade tumors having shorter potential doubling time [7]. In practice, it is unclear whether this effect is clinically relevant.

In this study, we report our experience introducing Cs-131 low dose rate brachytherapy for prostate cancer and compare with patients treated with I-125 and Pd-103. Given the retrospective nature of this study, the three groups were not matched in advance and the risk category for Cs-131 and I-125 cases was lower (Table 1). This is likely related to the fact that during the time period in which Pd-103 was used, generally higher risk cases were selected (Table 1). Fortunately, we found few PSA failures across all three isotope groups. It is notable the data suggests a lower rate of PSA failure for Cs-131 and a statistically significant difference compared to the Pd-103 and I-125 groups. However, this may be attributed to differences in risk categories between groups.

Clinical advantages for the Cs-131 isotope may increase the popularity of low dose rate brachytherapy for non-metastatic prostate cancers. The Ascende-RT trial [4] provided randomized data showing improved cancer control outcomes for intermediate and high-risk patients with the addition of low dose-rate brachytherapy to the combination of hormone therapy and external beam radiation. At a median follow-up of 6.5 years, it was noted that within the brachytherapy arm of the trial, there was increased rates of grade 3 GU events with 5 year cumulative incidence of $8.6 \%$ vs $2.2 \%(\mathrm{p}=0.058)$ and slightly greater grade $3 \mathrm{GI}$ morbidity $(8.1 \%$ vs $3.2 \%$; $\mathrm{p}=0.124)$. 
PSA levels at 3 and 6 months following treatment. The mean PSA for both groups was 7.3 pretreatment and initial post-treatment PSA was 1.5 in the I-125 group and 1.2 in the Cs-131 group.

Two recent large single arm studies have now confirmed the use of Cs-131 as an effective alternative isotope. Moran et al. [13] reported on 571 cases of Cs-131 prostate brachytherapy performed at the Prostate Cancer Foundation of Chicago. The five-year freedom from biochemical failure rates were $96.9 \%$ for low-risk, 92.8\% for intermediate-risk, and 93.2\% for high-risk patients, respectively. Similarly, Benoit et al. [14] reported on analysis of 669 men who had Cs-131 prostate implant at the University of Pittsburgh. Using the Phoenix criteria, biochemical freedom of disease was $97.1 \%$ at 5 years for low-risk, $94 \%$ for intermediaterisk, and $86.2 \%$ for high-risk cases. At 10 years, the rates were $95.3 \%, 90.1 \%$, and $56.6 \%$. In both groups, large numbers of intermediate and high-risk patients had combination treatment with brachytherapy and external beam radiotherapy. Although the current study is much smaller, we had similar biochemical control rates in the community hospital setting.

For our comparisons, the overall survival was greater for Cs-131 compared to Pd-103 and I-125 groups combined ( $\mathrm{p}=0.25$; Figure 2 ), but this was not statistically significant. This may be due to only 8 deaths in the Cs-131 group and shorter follow-up. It is our view that absolute differences in all-cause mortality here may be attributed to intercurrent disease. The overall survival in the Cs-131 group was similar to that reported by Moran et al. [13].

Finally, dosimetric comparison between isotopes revealed Cs-131 dosimetry qualitatively similar to I-125 and Pd-103. Prescription doses followed standard recommendations [15]. We found that for the same dose coverage of the prostate (D90), statistically greater V150 and V200 was observed using I-125 than Cs-131 (Figure 3). The delivered activity per seed and the number of seeds employed were greater compared to other isotopes. Corrected total source strength showed a similar distribution for Cs-131 compared to I-125 and Pd103 with mean percent V150 of 36\% for both I-125 and Pd-103 versus $27 \%$ for Cs-131 ( $<<0.0001)$ consistent with superior homogeneity with use of Cs-131. By comparison, Yang et al. [16] replanned cases previously implanted with I-125 using Cs-131 and Pd-103 prescriptions. They found that Cs-131 allowed for improved dose homogeneity with sparing of urethra and rectum and comparable or fewer seeds and needles required. This would suggest that Cs-131 may offer some dosimetric advantages compared with the other isotopes. It is clear that careful attention to patient selection and treatment planning will be important for clinical use of Cs-131 due to its high initial dose rate.

Permanent interstitial brachytherapy with or without external beam radiation is an attractive option for men presenting with non-metastatic prostate cancer. This study suggests similar PSA failure rates and overall survival for Cs-131 along with dosimetric equivalence compared to traditional isotopes [17,18].

This study has significant limitations which are related mainly to the fact that the patients were treated over a relatively long time period. We were not able to match the three groups and the risk category for the prostate cancer in the Cs-131 group was clearly lower. In the current analysis, we did not study the correlation between GU and GI toxicities and doses delivered to urethra and rectum. Other drawbacks of the study include relatively small numbers of cases, retrospective analysis, and shorter follow-up for PSA failure among the Cs-131 cases (27 months versus 40 months for I-125 and Pd-103). Nonetheless, with this experience in the community hospital setting, we continue to offer Cs-131 prostate brachytherapy alone or combined with external radiation and/or hormone therapy as a standard option based on a patients' risk category.

This work has previously been presented in part in abstract form at the 2020 American Society of Clinical Oncology Genitourinary Oncology Meeting and at the 2018 Michigan Cancer Consortium Annual Meeting $[17,18]$.

\section{Conclusions}

Permanent interstitial brachytherapy is an attractive option for select men presenting with low-risk and favorable intermediate-risk prostate cancer. This study compared three groups of patients treated with three different commonly used isotopes and represents the excellent outcomes that can be obtained in a community hospital system with these methods. There was excellent PSA control for Cs-131 patients similar to or better than I-125 and Pd-103 patients at 5 years. We also report that overall survival was not statistically different between the three groups of patients. Based on the shorter half-life, employing the Cs131 isotope may lead to a more rapid resolution of side-effects. For these reasons, we have changed our practice and offer Cs-131 based implants to most patients who are candidates for seed implants.

\section{Additional Information \\ Disclosures}

Human subjects: Consent was obtained or waived by all participants in this study. Ascension St John Hospital IRB issued approval 1036455-7. Review Details [1036455-7] Retrospective Analysis of Patients Treated with Radiotherapy for Prostate Cancer Ascension St. John Hospital IRB, Detroit, MI Submission 
Details Submitted To Ascension St. John Hospital IRB, Detroit, MI Submitted by Judith Boura Submission Date 01/05/2021 Submission Type Continuing Review/Progress Report Local Board Reference Number 1036455/172977 Review Details: Agenda Review Type Board Action Effective Date Project Status Expiration Date 01/21/2021 07:30 AM Expedited Review Approved 01/11/2021 Active 01/10/2022. Animal subjects: All authors have confirmed that this study did not involve animal subjects or tissue. Conflicts of interest: In compliance with the ICMJE uniform disclosure form, all authors declare the following: Payment/services info: All authors have declared that no financial support was received from any organization for the submitted work. Financial relationships: All authors have declared that they have no financial relationships at present or within the previous three years with any organizations that might have an interest in the submitted work. Other relationships: All authors have declared that there are no other relationships or activities that could appear to have influenced the submitted work.

\section{Acknowledgements}

The authors would like to acknowledge the contributions of the late Elayne Arterbery MD to this research and to the field of Radiation Oncology This work has previously been presented in part in abstract form at the 2020 American Society of Clinical Oncology Genitourinary Oncology Meeting (San Francisco, CA; reference 18) and at the 2018 Michigan Cancer Consortium Annual Meeting (Lansing, MI; reference 19).

\section{References}

1. U.S. Cancer Statistics Public Use Databases . (2021). Accessed: September 20, 2021: https://www.cdc.gov/cancer/uscs/public-use/index.htm.

2. NCCN Clinical Practice Guidelines in Oncology. Prostate Cancer: Version 1.2015 . (2021). Accessed: September 20, 2021: https://www.nccn.org/professionals/physician_gls/pdf/prostate.pdf.

3. Ennis RD, Hu L, Ryemon SN, Lin J, Mazumdar M: Brachytherapy-based radiotherapy and radical prostatectomy are associated with similar survival in high-risk localized prostate cancer. J Clin Oncol. 2018, 36:1192-8. 10.1200/JCO.2017.75.9134

4. Morris WJ, Tyldesley S, Rodda S, et al.: Androgen Suppression Combined with Elective Nodal and Dose Escalated Radiation Therapy (the ASCENDE-RT Trial): an analysis of survival endpoints for a randomized trial comparing a low-dose-rate brachytherapy boost to a dose-escalated external beam boost for high- and Intermediate-risk prostate cancer. Int J Radiat Oncol Biol Phys. 2017, 98:275-85. 10.1016/j.ijrobp.2016.11.026

5. Chin J, Rumble RB, Kollmeier M, et al.: Brachytherapy for patients with prostate cancer: American Society of Clinical Oncology/Cancer Care Ontario Joint Guideline Update. J Clin Oncol. 2017, 35:1737-43. 10.1200/JCO.2016.72.0466

6. Arterbery V, Chuba P, Sharma R, Caruthers J: Short term urinary morbidity following Cesium-131 ultrasound guided transperineal prostate seed implantation. Int J Radiat Oncol Biol Phys. 2010, 78:388 .

7. King CR, DiPetrillo TA, Wazer DE: Optimal radiotherapy for prostate cancer: predictions for conventional external beam, IMRT, and brachytherapy from radiobiologic models. Int J Radiat Oncol Biol Phys. 2000, 46:165-172. 10.1016/s0360-3016(99)00406-X

8. Ling DC, Chen KS, Benoit RM, Beriwal S, Smith RP: Long-term patient-reported rectal bleeding and bowelrelated quality of life after Cs-131 prostate brachytherapy. Int J Radiat Oncol Biol Phys. 2019, 104:622-30. 10.1016/j.ijrobp.2019.02.056

9. Blanchard P, Pugh TJ, Swanson DA, et al.: Patient-reported health-related quality of life for men treated with low-dose-rate prostate brachytherapy as monotherapy with 125-iodine, 103-palladium, or 131-cesium: results of a prospective phase II study. Brachytherapy. 2018, 17:265-276. 10.1016/j.brachy.2017.11.007

10. Rice SR, Olexa G, Hussain A, Mannuel H, Naslund MJ, Amin P, Kwok Y: A phase II study evaluating bone marrow-sparing, image-guided pelvic intensity-modulated radiotherapy (IMRT) with Cesium-131 brachytherapy boost, adjuvant chemotherapy, and long-term hormonal ablation in patients with high risk, nonmetastatic prostate cancer. Am J Clin Oncol. 2019, 42:285-91. 10.1097/COC.0000000000000520

11. Abramowitz MC, Li T, Buyyounouski MK, Ross E, Uzzo RG, Pollack A, Horwitz EM: The Phoenix definition of biochemical failure predicts for overall survival in patients with prostate cancer. Cancer. 2008, 112:55-60. 10.1002/cncr.23139

12. Tomaszewski JJ, Smaldone MC, Makaroun S, Smith RP, Beriwal S, Benoit R: Cesium 131 versus iodine 125 implants for prostate cancer: evaluation of early PSA response. Can J Urol. 2010, 17:5360-4.

13. Moran BJ, Rice SR, Chhabra AM, Amin N, Braccioforte M, Agarwal M: Long-term biochemical outcomes using cesium-131 in prostate brachytherapy. Brachytherapy. 2019, 18:800-5. 10.1016/j.brachy.2019.07.002

14. Benoit RM, Horne ZD, Houser CJ, Smith RP, Beriwal S: Cesium-131 prostate brachytherapy: A single institutional long-term experience. Brachytherapy. 2020, 19:298-304. 10.1016/j.brachy.2020.02.006

15. Rivard MJ, Butler WM, Devlin PM, et al.: American Brachytherapy Society recommends no change for prostate permanent implant dose prescriptions using iodine-125 or palladium-103. Brachytherapy. 2007, 6:34-7. 10.1016/j.brachy.2006.11.001

16. Yang R, Wang J, Zhang H: Dosimetric study of Cs-131, I-125, and Pd-103 seeds for permanent prostate brachytherapy. Cancer Biother Radiopharm. 2009, 24:701-5. 10.1089/cbr.2009.0648

17. Booher JG, Domenig P, Chuba PJ : Isotope selection and PSA failure in prostate seed implant: Experience with CS-131 in a community hospital system.. J Clin Oncol. 2020, 38:s375-375. 10.1200/JCO.2020.38.6_suppl.375

18. Booher JG, Boura J, Verdecchia K : Treatment of prostate cancer using Cesium-131 seed implant: dosimetric comparison with I-125. J Proc 2018 Michigan Cancer Consortium Annual Meeting. 2018. , 3:4-5. 